\title{
Familiarity and Attraction to Stimuli: Developmental Change or Methodological Artifact? ${ }^{1}$
}

\author{
ROBERT V. KAIL, JR. ${ }^{2}$ \\ University of Michigan, Ann Arbor
}

\begin{abstract}
One hundred thirteen 7-, 9-, and 11-year-old children viewed a series of stimuli consisting of Chinese characters exposed a different number of times. Following exposure to the stimuli, children ranked the stimuli according to liking. Seven- and 9-year-olds preferred the more familiar characters to those seen less frequently, but the oldest children preferred novel stimuli. In rankings taken 3 weeks after original exposure, a subsample of 11-yearolds preferred the more familiar characters. These results suggest that discrepancies obtained in previous research on the affective consequences of mere exposure are of methodological rather than developmental origin. Possible critical differences in procedure are discussed.
\end{abstract}

From a developmental perspective, recent research investigating the relationship between familiarity and attraction to a stimulus has produced ambiguous results. It is clear that the infant will avert his gaze from a familiar stimulus toward a novel one (Fantz, 1964). Similarly, in analyzing the effects of stimulus familiarization, Cantor et al. (Cantor, 1968, 1972; Faw \& Pien, 1971; Siebold, 1972) have found that children generally like novel stimuli more than familiar stimuli. However, on the basis of a series of experiments with adults (Crandall, Harrison \& Zajonc, 1974; Kail \& Freeman, 1973; Zajonc, 1968; Zajonc, Markus \& Wilson, 1974) it can be argued strongly that mere repeated exposure of an unfamiliar stimulus to an individual is sufficient to increase its attractiveness.

${ }^{1}$ This research was supported by NSF Grant GS 35495 to Robert B. Zajonc. The author was a predoctoral trainee on NIMH Grant No. 5 T01 MH-06667-14 while portions of this study were in progress. The author wishes to thank Harold Stevenson and John Hagen for their critical readings of drafts of this manuscript, and Gary Vosburg for his assistance in the collection of data. The author is particularly indebted to Robert B. Zajonc for his advice and assistance throughout this research. The principal, staff, and children of Spencer Elementary School, Whitmore Lake, MI, are gratefully acknowledged for their cooperation.

'Reprints should be requested from Robert V. Kail, Jr., Department of Psychology, University of Michigan, Ann Arbor, MI 48104. 
While it is possible that a developmental change in the exposure-affect relationship may occur between childhood and adulthood, there is no a priori reason to expect such a change. An alternative explanation would be that procedural differences between the paradigms used with children and with adults have produced the discrepant findings. This interpretation is supported by Berlyne's (1970) work with adults that has produced findings consistent with Cantor's research with children using similar methodology. Heingartner and Hall (1974), who used methodology more akin to that employed by Zajonc, found that children preferred more familiar music to less familiar selections following systematic exposure.

The present experiment was conducted to examine further the possibility that procedural differences may account for the ambiguous results obtained in previous research on the affective consequences of mere exposure. The experiment replicated one of Zajonc's (1968) early studies, with children as subjects. Stimuli were presented at different frequencies, and after exposure, subjects rank-ordered the stimuli according to liking. The stimuli were Chinese characters (from Zajonc, 1968). Children at three age levels used in previous research on the relationship between familiarity and attraction were tested to investigate the possibility of developmental changes in the exposure-affect relationship.

\section{METHOD}

\section{Subjects}

The subjects were 113 children, 56 boys and 57 girls, from Spencer Elementary School, Whitmore Lake, Michigan. All subjects were white, and lived in lower-middle to middle-class homes. They were divided into three groups by age (median ca: 7 years, 9 months; 9 years, 5 months; 11 years, 5 months). The children were tested in groups of three to six in a vacant classroom within the school. To equalize the size of the groups, the data of five randomly selected subjects were deleted.

\section{Stimuli}

Twelve Chinese characters were presented, with two each shown at $0,1,2,5,10$, and 25 exposures for a total presentation sequence of 86 slides. The stimuli were presented in two partially counterbalanced orders. Each stimulus was assigned a frequency of exposure for Order 1 and Order 2 reversed these frequencies. Thus, a character seen 25 times in Order 1 was exposed 0 times in Order 2; a character seen 10 times in Order 1 was exposed 1 time in Order 2. The order of presentation was random. 


\section{Procedure}

Subjects were told to watch some slides that would be shown to them. 'The exposure sequence was projected on a blank wall with a Kodak Carousel projector. Each slide was exposed for 2 sec, with the interstimulus interval the fraction of a second necessary to advance the projector. Following the exposure sequence, children rank-ordered the characters they had just seen according to how much they liked them. All children had a deck of 12 randomly ordered $3 \times 5$ in. index cards, each with a Xerox reproduction of one of the characters mounted on it. Subjects spread the cards before them on the desk top, selected their favorite card, and put it in a separate pile. From the remaining cards, subjects selected their second favorite card and placed it behind the card chosen previously. Subjects proceeded in this manner, with the assistance of the experimenter, until all 12 cards had been rank-ordered.

\section{RESULTS}

Because subjects rated two pictures exposed at each frequency, each subject generated two data points (ranks) for each of six frequencies of exposure. From these data, the slope of each subject's regression line (liking ranks regressed on $\log [$ frequency +1$]$ ) was calculated, as was the intercept. The average exposure function at each age, determined from the means of the slopes and intercepts, is presented in Fig. 1. Lower numbers (ranks) indicate greater liking. An analysis of variance computed on the slopes ${ }^{3}$ indicated a significant main effect only for age,

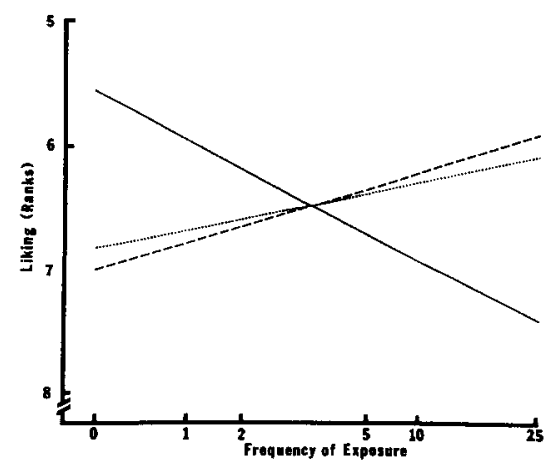

Fia. 1. The effect of frequency of exposure on liking (ranks), shown as average regression lines for 7-, 9-, and 11-year-old subjects. (- --7 -year-olds; -----9 -year-olds; 11-year-olds.)

${ }^{3}$ Because the slopes were computed from rank-order data, the intercepts are correlated perfectly with the slopes. Consequently, only the analyses of the slopes are presented here. 
TABLE 1

Number of Children at Each Agli Level with Positive and Negative Slopes

\begin{tabular}{cccccc}
\hline & \multicolumn{4}{c}{ Age } & Total \\
\cline { 3 - 5 } Type of slope & & 7 & 9 & 11 & 55 \\
\cline { 3 - 5 } \cline { 4 - 5 } Positive & & 21 & 11 & 11 & 53 \\
Negative & 15 & 13 & 25 & 53 \\
\hline
\end{tabular}

$F(2,96)=8.51, p<.001$. The combined exposure function of the $7-$ and 9 -year-olds had a positive slope $\left(\bar{X}_{7}=.72 ; \bar{X}_{9}=.61\right)$ significantly greater than zero, $t(\mathbf{7 1})=2.08, p<.05$, indicating increased liking as a function of exposure. For the 11-year-old subjects, extended exposure resulted in a strong negative slope $\left(\bar{X}_{11}=-1.30\right)$ reflecting decreased liking, $t(35)=3.68, p<.002$. Neuman-Keuls tests revealed significant differences between the 11-year-olds and the two younger groups, $p^{\prime}$ s $<.01$, but there was no significant difference between the 7 - and 9 -year-olds.

At each age level, subjects were divided into those with positive and negative slopes for the exposure functions. These data are presented in Table 1. These individual data confirm the findings of the grouped data, that most younger children preferred the more familiar to less familiar stimuli and the reverse was true in the oldest group, $\chi^{2}(2)=9.29, p<.01$.

\section{DISCUSSION}

The results of the 7 - and 9-year-old children are clearly consistent with the positive cxposurc-affect position, but the 11-ycar-olds' data resemble previous findings with children (Cantor, 1968). One explanation of these data is that an initially positive exposure-affect relation is replaced by a negative exposure function in late childhood, only to have the positive relationship reappear in adulthood. However, this interpretation does not explain the inconsistencies in previous research. Though few stimulus familiarization experiments have examined the effects of exposure across a developmental span, different experiments have been conducted with children of various ages. At all developmental levels, Cantor has noted the negative effects of stimulus exposure. Further, the children participating in the Heingartner-Hall experiment were 10year-olds, yet in that experiment a positive exposure-affect relationship obtained. Rather than a developmental change in the effects of exposure, it appears that some aspect of the procedure or task may have affected the older children differently.

Under some conditions of exposure, a decrease in liking is due to prop- 


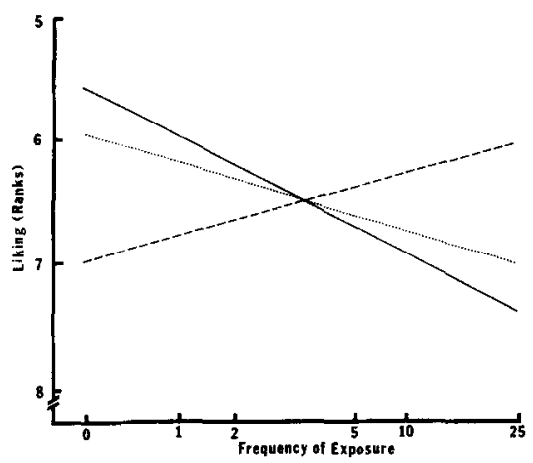

Fig. 2. The effect of frequency of exposure on immediate rankings for all 11-yearolds, on immediate rankings of a subsample of eight 11-year-olds, and on the delayed rankings of this subsample of 11 -year-olds, all shown as average regression lines. (See text for explanation.) (_- - Delayed Rankings for Subsample; - - - Immediate Rankings for Subsample; ___ Immediate Rankings.)

erties of the exposure situation and not to the number of exposures per se. Factors associated with the experimental task may causc lowercd ratings of stimuli when, in fact, the positive relationship between exposure and affect exists (Crandall et al., 1974; Kail \& Freeman, 1973). In the Crandall et al. experiment, adults' ratings immediately following exposure to a highly redundant exposure sequence indicated a preference for novel over more familiar stimuli. However, if ratings were delayed 1 or 4 weeks and the negative responses associated with the boring task thereby allowed to dissipate, the exposure-affect relationship again assumed a positive function. It is possible that the oldest children may have become bored with the experiment and reflected this boredom in their ratings of stimuli. Faw and Pien (1971) noted that their 10-, 11-, and 12-year-old subjects became disinterested in the experiment as it progressed, and consequently no differences attributable to exposure obtained.

To test this explanation of the negative exposure function obtained, a subsample of eight 11-year-olds ranked the stimuli a second time 3 weeks following exposure to stimuli. ${ }^{4}$ The average exposure function derived from these rankings is presented in Fig. 2, together with the initial functions. In the delayed rankings, a positive exposure function $(\bar{X}=.57)$ quite similar to the functions of the 7 - and 9-year-olds ob-

${ }^{4}$ Two groups of children were selected for second testing so that there was an equal number of boys and girls, as well as an equal number of children originally exposed to Orders 1 and 2. These variables were not completely counterbalanced in the delayed ranking data. Because subject anonymity was required in this experiment, no statistical comparisons can be made between the original and delayed ranking data. 
tained, in contrast to the original negative function. Apparently, the 11-year-olds were bored with the experiment, resulting in the negative exposure function. By the second rating 3 weeks later, the situational boredom had dissipated, and the exposure-affect relationship assumed a positive function.

This explanation is further supported by recent unpublished data (Kail, 1973). Children at the same three age levels were tested using procedures identical to those employed in the present study, except that the stimuli were photographs of children's faces. All age differences in the exposure functions were eliminated, and children at each age level demonstrated increased liking following exposure for faces of children of their own sex. Presumably, viewing photographs of faces was more interesting for the children than was watching the Chinese characters. Consequently when the task was interesting rather than boring for the older children, the 11-year-olds' data were quite consistent with those of the younger children and with adult data.

Siebold (1972) has presented data that are apparently inconsistent with the situational boredom hypothesis. Eleven-year-olds rated stimuli both immediately after the exposure series, and after delays of $5 \mathrm{~min}, 2$ or 7 days. In each case the unfamiliar stimuli were preferred to the familiar stimuli, and there was no indication of increased liking for the familiar stimuli as the length of the delay increased, as the situational boredom hypothesis would predict. Perhaps 1 week was insufficient time to allow such boredom to dissipate. However, these results may also have obtained because subjects rated stimuli during the exposure sequence as well as after it, a procedure that can result in a striking change in the shape of the exposure function, from positive to curvilinear or negative (Harrison \& Crandall, 1972).

Thus, the data presented in Figs. 1 and 2 provide supporting evidence for the proposition that procedural differences between the child and adult research have led to the ambiguities in the accumulated evidence, and underscore the distinction between affective responses to the stimuli and to the experimental task in general. There appear to be two additional critical differences that may influence the shape of the exposure function. One such difference involves the nature of the stimulus exposed to the subject. The positive exposure-affect relationship is a logarithmic function; most of the positive increments attributable to mere exposure occur during the initial exposures to a stimulus (Zajone, 1968). If the subject has been exposed to a stimulus frequently prior to the experiment (e.g., English words), a few additional exposures do not result in increased attraction (Amster \& Glasman, 1966). In much of the work with children (Cantor, 1968; Faw \& Pien, 1971; Siebold, 1972) subjects have been 
exposed to stimuli that are meaningful or familiar. Given the number of preexperimental exposures that many children have had to these stimuli, it is not surprising that a positive exposure effect did not obtain.

This difference would help to explain a perplexing finding from a recent Cantor (1972) experiment. In that study, 11-year-old white children rated faces of black boys seen frequently higher than those not seen prior to the rating sequence, but preferred faces of nonfamiliar whites over the familiar whites. Cantor indicated that the direct contact these white subjects had with black children was probably quite limited. Therefore, relative to faces of black children, faces of white children have had a great many more preexperimental exposures. The additional exposure in the familiarization phase may have resulted in enhanced liking for blacks because they are the only "truly novel" stimuli in the experiment for these subjects.

The response competition explanation of the exposure-affect relationship (Harrison, 1968) suggests an additional important difference in procedure. Novel stimuli elicit several responses based on their similarity to more familiar stimuli. Many of the responses may be mutually incompatible, creating a state of tension for the individual. Repeated exposure resolves this tension by reducing candidate responses and eventually associating a single response to the stimulus. Consequently, exposure reduces tension and leads to increased liking. In many studies with children (Cantor, 1972; Siebold, 1972), subjects were told prior to the exposure sequence that they would be rating the stimuli on various dimensions. This instructional set may have reduced response compctition and thus weakened the positive effects of exposure. ${ }^{5}$

Thus, the exposure effect is stable over a broad range of ages. Though not suggesting that subjects cannot become bored with repeated exposure, it is apparent that under conditions of mere exposure, children, like adults, prefer stimuli that they have seen frequently to those seen less frequently. Future research must delineate the critical procedures that differentiate the evidence supporting a positive exposure-affect relationship from research advocating a negative relationship.

\section{REFERENCES}

Amster, H., \& Glasman, L. D. Verbal repetition and connotative change. Journal of Experimental Psychology, 1966, 71, 389-395.

Berlyne, D. E. Novclty, complexity, and hedonic value. Perception \& Psychophysies, $1970,8,279-286$.

Cantor, G. N. Children's "like-dislike" ratings of familiarized and nonfamiliarized visual stimuli. Journal of Experimental Child Psychology, 1968, 6, 651-657.

${ }^{5}$ Rick Crandall originally suggested the importance of this difference. 
Cantor, G. N. Effects of familiarization on children's ratings of pictures of whites and blacks. Child Development, 1972, 43, 1219-1229.

Crandall, R., Harrison, A. A., \& Zajonc, R. B. The permanence of the positive and negative effects of stimulus exposure: A 'sleeper effect'?, Unpublished manuscript, 1974.

FAntz, R. L. Visual experience in infants: Decreased attention to familiar patterns relative to novel ones. Science, 1964, 146, 688-670.

FAW, T. T.. \& PiEn, D. The influence of stimulus exposure on rated preference: Effects of age, pattern of exposure, and stimulus meaningfulness. Journal of Experimental Child Psychology, 1971, 11, 339-346.

Harrison, A. A. Response competition, frequency, exploratory behavior, and liking. Journal of Personality and Social Psychology, 1968, 9, 363-368.

Harrison, A. A., \& Crandall, R. Heterogeneity-homogeneity of exposure sequence and the attitudinal effects of mere exposure. Journal of Personality and Social Psychology, 1972, 21, 234-238.

Heingartner, A., \& Hall, J. The affective consequences in adults and children of repeated exposure to auditory stimuli. Jownal of Personality and Social Psychology. 1974, 29, 719-723.

KaIL, R. V., JR., Familiarity and attraction to social and nonsocial stimuli. Unpublished manuscript, University of Michigan, Developmental Reports Series, 1973.

Kail, R. V., Jr., \& Freeman, H. R. Sequence redundancy, rating dimensions, and the exposure effect. Memory \& Cognition, 1973, 1, 454-458.

Stebold, J. R. Children's rating responses as related to amount and recency of stimulus familiarization and stimulus complexity. Journal of Experimental Child Psychology, 1972, 14, 257261.

Zajonc, R. B. Attitudinal effects of mere exposure. Journal of Personality and Social Psychology Monograph Supplement, 1968, 9, 1-27.

Zajonc, R. B., Markus, H., \& Wilson, W. R. Exposure effects and associative learning. Journal of Experimental Social Psychology. 1974, 10, 248-263. 